Published in final edited form as:

Circulation. 2015 August 18; 132(7): 578-585. doi:10.1161/CIRCULATIONAHA.115.016537.

\title{
Circulating Biomarkers and Abdominal Aortic Aneurysm Incidence: The Atherosclerosis Risk in Communities (ARIC) Study
}

\author{
Aaron R. Folsom, MD¹, Lu Yao, MD¹, Alvaro Alonso, MD, PhD ${ }^{1}$, Pamela L. Lutsey, PhD ${ }^{1}$, \\ Emil Missov, MD, $\mathrm{PhD}^{2}$, Frank A. Lederle, $\mathrm{MD}^{3}$, Christie M. Ballantyne, $\mathrm{MD}^{4}$, and Weihong \\ Tang, MD, PhD ${ }^{1}$ \\ ${ }^{1}$ Division of Epidemiology and Community Health, School of Public Health, University of \\ Minnesota, Minneapolis, MN \\ 2Division of Cardiology, Department of Medicine, School of Medicine, University of Minnesota, \\ Minneapolis, MN \\ ${ }^{3}$ Minneapolis VA Health Care System and Department of Medicine, School of Medicine, \\ University of Minnesota, Minneapolis, MN \\ ${ }^{4}$ Section of Cardiovascular Research, Department of Medicine, Baylor College of Medicine and \\ Center for Cardiovascular Disease Prevention, Houston Methodist DeBakey Heart and Vascular \\ Center, Houston, TX
}

\begin{abstract}
Background-The etiology of abdominal aortic aneurysm (AAA) is complex. Cross-sectional studies have connected circulating biomarkers with AAA, but prospective evidence is limited.

Methods and Results-In the Atherosclerosis Risk in Communities Study cohort, we measured multiple blood biomarkers of inflammation, hemostasis, thrombin generation, cardiac dysfunction, and vascular stiffness and identified incident AAAs during follow-up using hospital discharge codes. Six biomarkers (white blood cell count, fibrinogen, D-dimer, troponin T, Nterminal pro-brain natriuretic peptide, and high sensitivity C-reactive protein) were strongly positively associated with AAA incidence. Compared with having none of these six biomarkers in the highest quartile, the hazard ratios of AAA for those with 1, 2, 3, or 4-6 biomarkers in the highest quartile were 2.2,3.3,4.0, and 9.9, respectively ( $\mathrm{p}$ trend $<0.0001$ ), adjusted for other risk factors.
\end{abstract}

Conclusions-This prospective study found that higher concentrations of six biomarkers were associated with increased risk of AAA. The more markers that fell into the highest quartile, the higher the AAA risk. Multiple positive biomarkers identify a subgroup of patients at high risk of AAA.

\footnotetext{
Correspondence: Aaron R. Folsom, MD, Division of Epidemiology \& Community Health, School of Public Health, University of Minnesota, 1300 South $2^{\text {nd }}$ Street, Suite 300, Minneapolis, MN 55454, Phone: 612-626-8862, Fax: 612-624-0315, folso001@umn.edu.

Disclosures: None of the other authors declared a conflict of interest.
} 


\section{Keywords}

abdominal aortic aneurysm; risk factor; prospective cohort study

Epidemiologic studies have identified several risk factors for abdominal aortic aneurysms (AAA). The most important AAA risk factors are advanced age, male sex, white race, smoking, and family history, with hypertension and hypercholesterolemia playing less important roles. ${ }^{1}$ Diabetes is associated with reduced AAA risk. ${ }^{1}$ The pathophysiology of AAA involves infiltration of the aortic wall by inflammatory and immune cells, proteolysis of elastin and collagen in the media and adventitia, and smooth muscle cell apoptosis with thinning of the media. ${ }^{1}$ Several studies have identified circulating inflammatory, hemostatic, proteolytic, or extracellular matrix biomarkers associated with AAA presence or progression. ${ }^{2}$ Identification of biomarker associations may offer clues to AAA pathogenesis, aid in AAA diagnosis or prognosis assessment, or possibly help identify treatment targets.

Most previous AAA biomarker studies have been cross-sectional ${ }^{2}$ and therefore unable to address whether biomarker elevations precede or are etiologically important to AAA, versus being a consequence of AAA or a simple epiphenomenon in a complex disease process. Few studies have examined multiple biomarkers simultaneously. Therefore, more prospective, population-based evidence for biomarkers of inflammation, hemostasis, thrombin generation, cardiac dysfunction, and vascular stiffness related to AAA could be useful. Focusing on already measured biomarkers in those domains in the Atherosclerosis Risk in Communities (ARIC) cohort, we examined the association of multiple circulating biomarkers with incident AAA.

\section{Methods}

\section{Study Population}

The ARIC Study ${ }^{3}$ recruited a population-based cohort of 15,792 persons between 45 and 64 years of age in 1987 through 1989, from Forsyth County, North Carolina; Jackson, Mississippi (African Americans only); the northwest suburbs of Minneapolis, Minnesota; and Washington County, Maryland. ARIC performed a baseline examination of participants and followed them by annual telephone contact and up to four reexaminations in 19901992, 1993-1995, 1996-1998, and 2011-2013. We conducted follow-up for clinical AAAs after baseline and ascertainment of asymptomatic AAAs by an ultrasound screen in 201113, as shown in the study design (Supplemental Figure 1).

We proposed this research on biomarkers and AAA in an R01 grant proposal in 2010, which was funded by the National Institutes of Health. We proposed to study these specific biomarkers and AAA, a priori, in an ARIC manuscript proposal in 2014.

\section{Baseline (1987-89) Measurements}

ARIC staff drew blood after an 8-hour fasting period with minimal trauma from an antecubital vein. A central laboratory measured hemostatic variables from citrated plasma, which had been stored at $-70^{\circ} \mathrm{C}$ for a few weeks. ${ }^{4,5}$ In brief, the laboratory measured 
fibrinogen by the thrombin-time titration method ${ }^{6}$ with reagents and calibration materials (Fibriquik) obtained from General Diagnostics (Organon-Technika Co.); Factor VII and VIII activities by determining the ability of the tested sample to correct the clotting time of human factor VII- or factor VIII-deficient plasma obtained from George King Biomedical Inc.; von Willebrand factor antigen and protein $\mathrm{C}$ antigen using ELISA kits from American Bioproducts Company; anti-thrombin III (AT-III) activity by a chromogenic substrate method; and activated partial thromboplastin time (aPTT) on an automated coagulometer (Coag-A-Mate X-2, General Diagnostics). The reference material for assays was the Universal Coagulation Reference Plasma (Thromboscreen, Pacific Hemostasis, Curtin Matheson Scientific, Inc.). Reliability coefficients obtained from repeated testing of individuals over several weeks were 0.72 for fibrinogen, 0.78 for factor VII, 0.86 for factor VIII, 0.68 for von Willebrand factor, 0.56 for protein C, 0.42 for AT-III, and 0.92 for aPTT. $^{7}$

Laboratories in each study community measured white blood cell count (WBC) using Coulter counters. A central laboratory measured plasma total cholesterol ${ }^{8}$ by enzymatic methods and calculated LDL cholesterol. ${ }^{9}$ The laboratory measured HDL cholesterol after dextran-magnesium precipitation of non-HDL lipoproteins. ${ }^{10}$ ARIC defined prevalent diabetes mellitus as a fasting glucose level $\geq 126 \mathrm{mg} / \mathrm{dL}$, nonfasting glucose level $\geq 200$ $\mathrm{mg} / \mathrm{dL}$, a history of diabetes, or treatment for diabetes.

ARIC staff measured weight and height with participants in scrub suits. Staff took three blood pressure measures with a random-zero sphygmomanometer, and we averaged the last two measurements for analysis. ARIC collected information about use of anticoagulants from the medication bottles brought to the visit.

\section{Additional Biomarkers from 1993-1995 and 1996-1998}

Using fasting citrate plasma that had been collected at visit 3 (1993-1995) and stored unthawed at $-70^{\circ} \mathrm{C}$ until analysis in 2014 , ARIC measured D-dimer concentrations via an immuno-turbidimetric assay (Liatest D-DI; Diagnostica Stago, Parsippany, NJ) on the Evolution analyzer (Diagnostica Stago, Parsippany, NJ). The normal reference range is 0.22 $-4.0 \mu \mathrm{g} / \mathrm{mL}$, with expected normal values $<0.4 \mu \mathrm{g} / \mathrm{ml}$. Blind analysis of 73 pairs of ARIC samples split at the time of blood draw and stored until 2014 yielded an intra-class reliability coefficient of 0.92 .

Using EDTA plasma samples stored at $-70^{\circ} \mathrm{C}$ at visit 4 (1996-1998), ARIC measured troponin T (cTnT), $N$-terminal pro-brain natriuretic peptide (NT-proBNP), and high sensitivity C-reactive protein (CRP). The laboratory measured cardiac cTnT on a Cobas e411 analyzer using the Elecsys Troponin T immunoassay (Roche Diagnostics, Indianapolis, $\mathrm{IN})$. The limit of measurement is $3 \mathrm{ng} / \mathrm{L}$. The reliability coefficient for blinded quality control replicate measurements ( $n=418$ pairs) of cTnT from single blood draws was 0.98 . The ARIC laboratory measured plasma NT-proBNP on a Cobas e411 analyzer using the Elecys proBNP II immunoassay (Roche Diagnostics). The measuring range of the assay is 5 to $35,000 \mathrm{pg} / \mathrm{mL}$. The reliability coefficient for blinded replicate measurements was 0.99 ( $n$ $=418$ pairs). ARIC measured CRP by the immunoturbidimetric CRP-Latex (II) high sensitivity assay from Denka Seiken (Tokyo, Japan) using a Hitachi 911 analyzer (Roche 
Diagnostics). The reliability coefficient for blinded replicate measurements was 0.99 ( $n=55$ pairs).

\section{Ascertainment of AAA}

At the baseline examination in 1987-89, ARIC did not query the 45-64 year old participants about AAA history, but asked extensively about any prior arterial surgery. For this analysis of incident AAA, we excluded participants reporting prior AAA surgery or aortic angioplasty.

ARIC identified incident AAAs by several strategies. In the annual telephone calls with ARIC participants, interviewers asked about any interim hospitalizations and identified deaths, and these records were sought. ARIC also conducted surveillance of local hospitals to identify additional hospitalizations or deaths. In addition, ARIC linked participant identifiers with fee-for-service Medicare data from the Centers for Medicare and Medicaid Services (CMS) for 1991-2011, to find any missing hospital or outpatient events for those over 65 years. We identified clinical AAAs as those with a hospital discharge diagnosis from any source, or two Medicare outpatient claims that occurred at least one week apart, with ICD-9-CM codes of 441.3 (ruptured AAA), 441.4 (AAA without mention of rupture) or 441.02 (AAA dissection), or procedure codes of 38.44 (AAA resection and replacement) or 39.71 (AAA endovascular repair), or a listed cause of death coded as ICD-9 441.3 or 441.4 or $I C D$-10 code I71.02 (AAA dissection), I71.3 (ruptured AAA), or I71.4 (AAA without mention of rupture). Although labeled "clinical AAAs" these diagnoses would include both symptomatic and asymptomatic AAAs that were medically documented. We treated thoracic, thoracoabdominal, or unspecified aortic aneurysms as non-events.

To identify additional asymptomatic AAAs in the surviving ARIC cohort in 2011-2013, we performed a screening abdominal ultrasound in the fifth ARIC examination. A physician and a technician specializing in vascular imaging centrally trained experienced cardiac ultrasonographers from each ARIC site in abdominal scanning. After certification, the sonographers obtained aortic images with a Philips iE33 high resolution duplex scanner, using a Philips C5-1 transducer (Philips Healthcare, Bothell, WA). They recorded images of anterior-posterior and transverse diameters at the proximal aorta just below the superior mesenteric artery, the proximal infrarenal aorta $2 \mathrm{~cm}$ below the renal arteries, the distal infrarenal aorta $1 \mathrm{~cm}$ above the bifurcation, and the point of maximal infrarenal aortic diameter. They also recorded a longitudinal view of the infrarenal aorta. To identify all AAAs $\geq 3 \mathrm{~cm}$, vascular imaging physicians reviewed any image that the sonographers judged had $>2.8 \mathrm{~cm}$ maximal infrarenal diameter or probable pathology, plus a $5 \%$ random sample of the rest. Of the 10,036 ARIC participants still alive through August 2013, 6,538 $(65 \%)$ had a home or clinic ARIC examination and, of these, 5,911 (59\%) had usable abdominal ultrasonograms.

\section{Data Analysis}

The majority of measured biomarkers were from ARIC baseline, and so we describe the analysis from baseline. For markers measured at later ARIC visits, we performed an 
analogous analysis using covariates and exclusions for that visit. We used SAS, Version 9.3 (SAS Institute, Inc., Cary, NC) for analyses.

From the 15,792 ARIC participants, we excluded at baseline the few participants who were not white or African American or were African American in Minneapolis or Washington County $(n=103)$, those who were taking anticoagulants $(n=89)$, had prior AAA surgery $(n$ $=10)$, or had all biomarkers missing $(n=151)$. We also excluded those whose follow-up AAA status was uncertain $(n=28)$, leaving 15,411 at risk of AAA. We calculated length of follow-up as the time elapsed from the baseline examination to the first clinical AAA event, date of death, date of last contact (if lost), or through December 31, 2011. We first explored the association between each biomarker and incident AAA via cubic-spline graphs (not shown here). Ultimately, we grouped biomarkers into quartiles based on their overall distributions. We computed incidence rates of clinical AAA within quartiles and adjusted for age, race, and sex (Model 1) using Poisson regression models.

We performed multivariate modeling to obtain hazard ratios of incident clinical AAA in relation to biomarker quartiles by use of Cox proportional hazards regression. We tested for trend in hazard ratios across quartiles using an ordinal variable designating each quartile. We adjusted in Model 2 for clinical AAA risk factors: age, sex, race (African American, white), ARIC field center, total cholesterol, HDL cholesterol, use of lipid medication (yes, no), systolic blood pressure, antihypertensive medication use (yes, no), diabetes (yes, no), pack-years of cigarettes smoked, height, and weight. For this model, we tested whether the proportional hazards assumption held for the six main biomarkers, using interaction terms with the logarithm of follow-up time; the assumption held for all but a minor violation for D-dimer. Because sex and smoking status (ever, never) are key AAA risk factors, we also performed subgroup analyses stratified by these variables and tested for multiplicative interactions by including cross-product terms in the models. Finally, we created a multiple biomarker score by summing the number of biomarkers that were in the highest quartile.

We used the ultrasound-detected new AAAs from ARIC visit 5 in 2011-2013 to verify our main findings on risk factors for incident clinical AAAs. From 5,911 ARIC participants with usable ultrasounds, we excluded participants who were not white or African American $(n=$ 39 ), users of anticoagulants ( $n$ varied by visit the biomarker was measured), those with a clinical AAA before visit $5(n=100)$, and those with no prior biomarkers measured $(n=7)$. Depending on the biomarker, the analyses included 5,183 to 5,707 participants in whom we identified 64 to 74 asymptomatic AAAs. To adjust for the potential selection bias caused by differential participation in the 2011-2013 exam, we used inverse probability of attrition weighting (IPAW), as previously described. ${ }^{11}$ Briefly, for each participant we calculated weights based on the product of the probability of being alive at visit 5 and the probability of having an abdominal ultrasound conditional on being alive given covariates measured at baseline (sex, race, age, education, diabetes, hypertension, prevalent coronary heart disease, prevalent peripheral artery disease, prevalent heart failure, prevalent stroke, study site, smoking status, pack-years, physical activity, body mass index, HDL cholesterol, LDL cholesterol, total cholesterol, blood glucose, height, alcohol intake, white blood cell count, fibrinogen) and during follow-up (incident myocardial infarction, heart failure, or stroke). We obtained odds ratios and 95\% confidence intervals from IPAW-weighted general 
estimating equation models using a logit link and robust standard errors, ${ }^{11}$ which incorporated the added uncertainty of the weights in this analysis.

\section{Results \\ Descriptive Data}

At baseline in 1987-89, the 15,411 ARIC participants at risk of developing AAA were 4564 years old, $45 \%$ were men, $74 \%$ were whites, $12 \%$ had diabetes, $25 \%$ used antihypertensive medication, and $58 \%$ had ever smoked. Other participant characteristics at baseline and biomarker concentrations at the ARIC visits are shown in Supplemental Table 1. From baseline through 2011, over a median of 22.5 years of follow-up, we identified 587 clinical AAAs.

\section{Association of Individual biomarkers with Clinical AAA}

As shown in Table 1, after adjustment for age, race, and sex (Model 1), baseline fibrinogen and WBC count were strongly positively associated with incident clinical AAA, with hazard ratios approximately 3-fold higher in the highest versus lowest quartiles. With further adjustment for other AAA risk factors (Model 2), the AAA associations with fibrinogen and WBC count remained strong. In sex-specific analyses (involving 418 AAAs in men and 165 in women), the Model 2 trends remained highly significant ( $p$ trend $<0.005$ ) for WBC count and fibrinogen (Figure 1), and the hazard ratios of AAA for the highest quartiles were similar in both men and women. In smoking-specific analyses (involving 506 AAAs in ever smokers and 71 in never smokers, Figure 2), the fibrinogen association with AAA was significant for ever smokers and nearly so for never smokers. The association of WBC count with AAA was present for ever smokers and not for never smokers ( $\mathrm{p}$ interaction $=0.07$ ).

The associations of AAA incidence with baseline factor VII, factor VIII, von Willebrand factor, protein C, antithrombin III, and aPTT were nonexistent or weak (Supplemental Table 2).

Among 12,887 participants at ARIC visit 3, 12,271 were at risk and had D-dimer data. We identified 431 AAAs over a median of 16.8 years of follow-up. D-dimer was positively associated with incident AAA, with hazard ratios over 3.7 for the highest versus lowest quartiles (Table 2). The D-dimer associations with AAA were similar (p interaction >0.05) by sex (Figure 1) and for ever smokers and never smokers (Figure 2).

Among 11,656 participants at ARIC visit 4, 10,964 were at risk for AAA and had biomarkers measured. We identified 362 AAAs over a median of 13.9 years of follow-up. CRP, cTnT, and NT-pro BNP were all positively associated with incident AAA in both Models 1 and 2, with hazard ratios approximately 2-fold higher for the highest versus lowest quartiles (Table 3). As shown in Figures 1 and 2, the associations were similar for men and women and for ever smokers and never smokers (all p interaction $p>0.20$ ), even though Figure 2 clearly shows that CRP was not associated with AAA in never smokers (and the lack of a significant CRP by smoking interaction was likely due to low precision as only 51 AAAs occurred in never smokers after visit 4). 


\section{Association of Multiple Biomarker Score with Clinical AAA}

Using the six biomarkers strongly associated with AAA (i.e., WBC count, fibrinogen, Ddimer, cTnT, NT-proBNP, and CRP), we created a score (ranging 0-6) that summed for each participant the number of biomarkers in the highest quartile. After we excluded those with any missing biomarkers, those with AAA prior to visit 4 , those using anticoagulants, and those with any missing Model 2 covariates at visit 4, there were 8,876 visit 4 participants at risk of AAA. After visit 4, we identified 285 incident AAA events. As shown in Figure 3, there was a nearly 10-fold gradient in AAA incidence across categories of the biomarker score; Model 2 hazard ratios were 1, 2.2, 3.3, 4.0, and 9.9 for having 0, 1, 2, 3, and 4-6 elevated biomarkers, respectively ( $p$ trend $<0.0001$ ). The number of elevated biomarkers remained strongly associated with AAA incidence in a graded manner when we repeated this analysis excluding participants who had an ARIC medical history of myocardial infarction, stroke, or heart failure (hazard ratios 1, 2.2, 3.3, 3.8, 9.9; $\mathrm{p}$ trend < $0.0001)$

\section{Association of Biomarkers with Ultrasound AAAs}

Using ultrasound screening among ARIC visit 5 participants with no history of clinical AAA by 2011-2013, we identified only 64 to 74 additional AAAs, depending on the biomarker being considered (Table 4). WBC count and fibrinogen were strongly positively associated with asymptomatic AAA, whereas D-dimer, cTnT, NT-proBNP, and CRP were not.

\section{Discussion}

This population-based study found strong positive associations of clinical AAA incidence with circulating biomarkers of inflammation (CRP, WBC count, fibrinogen), thrombin generation (D-dimer), increased cardiac pressure and vascular stiffness (NT-proBNP), ${ }^{12,13}$ and cardiac injury (cTnT). These associations were independent of other major AAA risk factors and were mostly similar for men and women and for ever smokers and never smokers. The relation of AAA risk across quartiles of the significant biomarkers tended to be "graded", and this was confirmed by cubic spline plots. Moreover, the elevated AAA risk for the highest quartiles was generally at levels well below clinically relevant cutpoints for WBC count, D-dimer, NT-proBNP, and cTnT. The more markers a person had in the highest quartile, the greater the AAA risk, so that participants with 4-6 biomarkers elevated had a nearly 10-fold higher risk than those with no biomarkers elevated. The fact that our study was prospective, with long follow-up, suggests that circulating biomarkers are indicative of patients at high AAA risk, well before the clinical recognition of AAA.

We also confirmed that WBC count and fibrinogen were risk markers for subclinical AAA detected by ultrasound, whereas D-dimer, cTnT, NT-proBNP, and CRP were not. However, this analysis involving $\leq 74$ AAAs had limited power.

The observed associations offer support for multiple pathways in the etiology of AAA. Previous studies, mostly cross-sectional, have found similar positive associations of AAA with circulating levels of CRP, fibrinogen, and other inflammatory markers like interleukin- $6 .^{2}$ Moreover, a candidate gene study linked a CRP polymorphism with AAA, ${ }^{14}$ 
and a Mendelian randomization study implicated interleukin-6 pathways in AAA. ${ }^{15}$ These genetic studies, coupled with pathologic evidence, ${ }^{1}$ support a causal role of inflammation in AAA development. Our observation and that of Iribarren et al, ${ }^{16}$ that higher WBC count is associated with greater AAA incidence, is also consistent with a role of inflammation in AAA etiology.

Intraluminal thrombus is often present in AAAs, suggesting that increased thrombogenic factors may also play an etiologic role. We found that the thrombosis marker, D-dimer, was associated positively with AAA incidence, consistent with several cross-sectional studies. ${ }^{2}$ Thus, asymptomatic adults with greater than average D-dimer levels, possibly reflecting microthrombi, appear to be at increased risk of subsequent AAA. Other hemostatic markers associated with atherosclerotic or venous thrombotic events in ARIC ${ }^{17-20}$ showed little association with AAA.

Our most novel findings were the strong positive associations of AAA with NT-proBNP, a marker of increased cardiac filling pressure and vascular resistance, ${ }^{12,13}$ and with cTnT, a marker of myocardial injury. A previous cross-sectional study reported that NT-proBNP was correlated positively with aortic diameters in women. ${ }^{21}$ In the general ARIC population, the positive association between NT-proBNP and AAA probably reflects a role for increased systemic vascular resistance in AAA and a secondary rise in cardiac biomarkers. In any case, adults with greater NT-proBNP and cTnT levels are seemingly at increased risk of AAA, as part of their generally high risk of other cardiovascular diseases. ${ }^{22,23}$

Most associations between biomarkers and AAA (except for WBC count and CRP) were qualitatively similar for ever smokers and never smokers, although often not quite statistically significant for never smokers due to few AAAs among them. Because smoking is such a strong risk factor for AAA, it is difficult to control for its confounding effect by regression modeling. Demonstration of positive associations between some biomarkers and AAA, in never smokers, makes the observed associations more believable. Yet, the absence of an association of AAA in never smokers with two strong markers of inflammation (WBC count and CRP) suggests residual confounding by smoking still may affect our results.

Our study was large and population-based, but its assessment of clinical AAAs was limited to hospital and death ICD codes. Although we do not have direct evidence of the validity of ICD codes for AAA, the AAA codes seem quite specific, yet are likely insensitive for capturing events. We tried to enhance the validity of our AAA outcome by excluding aortic aneurysms coded to uncertain sites, as they may not have been abdominal. The ICD codes we used should include symptomatic AAAs, but also include some asymptomatic AAAs in the category "AAA without mention of rupture." In the absence of a physical or radiological screen for AAA, using ICD codes could miss some asymptomatic AAAs. We could not perform routine AAA screening throughout ARIC follow-up, but we were able to do a single ultrasound screening in approximately 60\% of living ARIC participants in 2011-2013. Relatively few silent AAAs were detected in 2011-2013, but this would not address asymptomatic AAAs missed in those who had died or were not screened. In general, any misclassification of AAA status is likely to be unrelated to biomarker levels and therefore would lead to our hazard ratio estimates being underestimates. 
While our study had many strengths, additional drawbacks warrant consideration. Firstly, the circulating biomarkers were measured just once. Some were measured soon after the baseline ARIC visit; others were measured on stored plasma samples at later examinations. Any biologic variability or change over time in the biomarkers most likely would attenuate the reported hazard ratios. Secondly, although we adjusted for several other major AAA risk factors, the observed associations may still suffer from residual confounding. In particular, we could not consider markers of extracellular matrix (e.g., procollagen) or its degradation (e.g., elastase or matrix metaloproteinases), and by not accounting for these, our hazard ratios may have been over-estimated. We have plans to measure several of these markers in an ARIC nested case-control study. Thirdly, although we excluded the few ARIC participants with AAA repair prior to baseline, without baseline ultrasound screening it is likely that some participants with prevalent AAAs remained in the "at risk" cohort. However, given the original 45-64 year ARIC age span, the number of prevalent AAAs should have been low. Fourthly, given just one ultrasound assessment at ARIC visit 5, we could not directly assess whether these biomarkers predict progression from mild aortic dilation to frank AAA. Thus, despite documenting prospectively that higher circulating biomarkers are associated with increased AAA incidence, our study does not establish a cause-effect relation or the clinical utility of measuring these biomarkers for AAA prediction.

In conclusion, this prospective study found that higher concentrations of CRP, WBCs, fibrinogen, D-dimer, cTnT, and NT-proBNP were associated with increased risk of AAA. The more markers that fell into the highest quartile, the higher the AAA risk. Multiple positive biomarkers identify a subgroup of patients at high risk of AAA.

\section{Supplementary Material}

Refer to Web version on PubMed Central for supplementary material.

\section{Acknowledgments}

The authors thank the staff and participants of the ARIC Study for their important contributions, and Drs. Carol Steenson and David Hunter for ultrasound reading.

Funding Sources: The National Heart, Lung, and Blood Institute (NHLBI) supported this research through R01 HL103695, U01 HL096902, and ARIC contracts HHSN268201100005C, HHSN268201100006C, HHSN268201100007C, HHSN268201100008C, HHSN268201100009C, HHSN268201100010C, HHSN268201100011C, and HHSN268201100012C.

Dr. Ballantyne has received research grants from Roche and provisional patent (patent no. 61721475) entitled Biomarkers to Improve Prediction of Heart Failure Risk filed by Baylor College of Medicine and Roche.

\section{References}

1. Nordon IM, Hinchliffe RJ, Loftus IM, Thompson MM. Pathophysiology and epidemiology of abdominal aortic aneurysms. Nat Rev Cardiol. 2011; 8:92-102.

2. Golledge J, Tsao PS, Dalman RL, Norman PE. Circulating markers of abdominal aortic aneurysm presence and progression. Circulation. 2008; 118:2382-2392. [PubMed: 19047592]

3. ARIC Investigators. The Atherosclerosis Risk in Communities (ARIC) Study: design and objectives. Am J Epidemiol. 1989; 129:687-702. [PubMed: 2646917] 
4. Papp AC, Hatzakis H, Bracey A, Wu KK. ARIC hemostasis study, I: development of blood collection and processing system suitable for multicenter hemostatic studies. Thromb Haemost. 1989; 61:15-19. [PubMed: 2526384]

5. Folsom AR, Wu KK, Rosamond WD, Sharrett AR, Chambless LE. Prospective study of hemostatic factors and incidence of coronary heart disease: The Atherosclerosis Risk in Communities (ARIC) Study. Circulation. 1997; 96:1102-1108. [PubMed: 9286936]

6. Clauss A. Gerinnungsphysiologische Schnellmethode zur Bestimmung des Fibrinogens. Acta Haematol. 1957; 17:237-246. [PubMed: 13434757]

7. Chambless LE, McMahon R, Wu K, Folsom A, Finch A, Shen YL. Short-term intra-individual variability in hemostasis factors: The ARIC Study. Ann Epidemiol. 1992; 2:723-733. [PubMed: 1342324]

8. Siedel J, Hegele EO, Ziegenhorn J, Wahlefeld AW. Reagent for the enzymatic determination of total serum cholesterol with improved lipolytic efficiency. Clin Chem. 1983; 29:1075-1080. [PubMed: 6851096]

9. Friedewald WT, Levy RI, Fredrickson DS. Estimation of the concentration of low-density lipoprotein cholesterol in plasma, without use of the preparative ultracentrifuge. Clin Chem. 1972; 18:499-502. [PubMed: 4337382]

10. Warnick GR, Benderson JM, Albers JJ. Quantitation of high-density-lipoprotein subclasses after separation by dextran sulfate and $\mathrm{Mg}^{2+}$ precipitation [Abstract]. Clin Chem. 1982; 28:1574.

11. Gottesman RF, Rawlings AM, Sharrett AR, Albert M, Alonso A, Bandeen-Roche K, Coker LH, Coresh J, Couper DJ, Griswold ME, Heiss G, Knopman DS, Patel MD, Penman AD, Power MC, Seines OA, Schneider AL, Wagenknecht LE, Windham BG, Wruck LM, Mosley TH. Impact of differential attrition on the association of education with cognitive change over 20 years of followup: the ARIC Neurocognitive Study. Am J Epidemiol. 2014; 179:956-966. [PubMed: 24627572]

12. Coutinho T, Turner ST, Mosley TH, Kullo IJ. Biomarkers associated with pulse pressure in African-Americans and non-Hispanic whites. Am J Hypertens. 2012; 25:145-151. [PubMed: 22012208]

13. Chen YC, Lee MC, Lee CJ, Ho GJ, Yin WY, Chang YJ, Hsu BG. N-terminal pro-B-type natriuretic peptide is associated with arterial stiffness measures using the cardio-ankle vascular index in renal transplant recipients. J Atheroscler Thromb. 2013; 20:646-653. [PubMed: 23665842]

14. Saratzis A, Bown M, Wild B, Sayers RD, Nightingale P, Smith J, Johnson C, Kitas G. C-reactive protein polymorphism rs3091244 is associated with abdominal aortic aneurysm. J Vasc Surg. 2014; 60:1332-1339. [PubMed: 24135623]

15. Harrison SC, Smith AJ, Jones GT, Swerdlow DI, Rampuri R, Bown MJ, on behalf of the Aneurysm Consortium. Folkersen L, Baas AF, de Borst GJ, Blankensteijn JD, Price JF, van der Graaf Y, McLachlan S, Agu O, Hofman A, Uitterlinden AG, Franco-Cereceda A, Ruigrok YM, van't Hof FN, Powell JT, van Rij AM, Casas JP, Eriksson P, Holmes MV, Asselbergs FW, Hingorani AD, Humphries SE. Interleukin-6 receptor pathways in abdominal aortic aneurysm. Eur Heart J. 2013; 34:3707-3716. [PubMed: 23111417]

16. Iribarren C, Darbinian JA, Go AS, Fireman BH, Lee CD, Grey DP. Traditional and novel risk factors for clinically diagnosed abdominal aortic aneurysm: the Kaiser Multiphasic Health Checkup Cohort Study. Ann Epidemiol. 2007; 17:669-678. [PubMed: 17512215]

17. Folsom AR, Wu KK, Rosamond WD, Sharrett AR, Chambless LE. Prospective study of hemostatic factors and incidence of coronary heart disease: The Atherosclerosis Risk in Communities (ARIC) Study. Circulation. 1997; 96:1102-1108. [PubMed: 9286936]

18. Tsai AW, Cushman M, Rosamond WD, Heckbert SR, Tracy R, Aleksic N, Folsom AR. Coagulation factors, inflammation markers, and venous thromboembolism: The Longitudinal Investigation of Thromboembolism Etiology (LITE). Am J Med. 2002; 113:636-642. [PubMed: 12505113]

19. Folsom AR, Aleksic N, Wang L, Cushman M, Wu KK, White RH. Protein C, antithrombin, and venous thromboembolism incidence: A prospective population-based study. Arterioscler Thromb Vasc Biol. 2002; 22:1018-1022. [PubMed: 12067914] 
20. Zakai NA, Ohira T, White R, Folsom AR, Cushman M. Activated partial thromboplastin time and risk of future venous thromboembolism. Am J Med. 2008; 121:231-238. [PubMed: 18328308]

21. Gutin LS, Bakalov VK, Rosing DR, Arai AE, Gharib AM, Bondy CA. N-terminal pro-brain natriuretic peptide levels and aortic diameters. Am Heart J. 2012; 164:419-424. [PubMed: 22980310]

22. Folsom AR, Nambi V, Bell EJ, Oluleye OW, Gothesman RF, Lutsey PL, Huxley RR, Ballantyne CM. Troponin T, N-terminal pro-B-type natriuretic peptide, and incidence of stroke: The Atherosclerosis Risk in Communities Study. Stroke. 2013; 44:961-967. [PubMed: 23471272]

23. Ye Z, Ali Z, Klee GG, Mosley TH Jr, Kullo IJ. Associations of candidate biomarkers of vascular disease with the ankle-brachial index and peripheral arterial disease. Am J Hypertens. 2013; 26:495-502. [PubMed: 23467205] 
WBC count

Fibrinogen

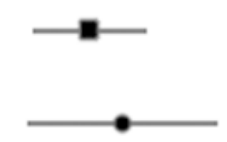

- Men

D-dimer

Troponin T

NT proBNP

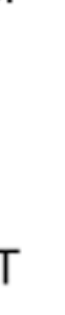

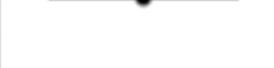

- Women

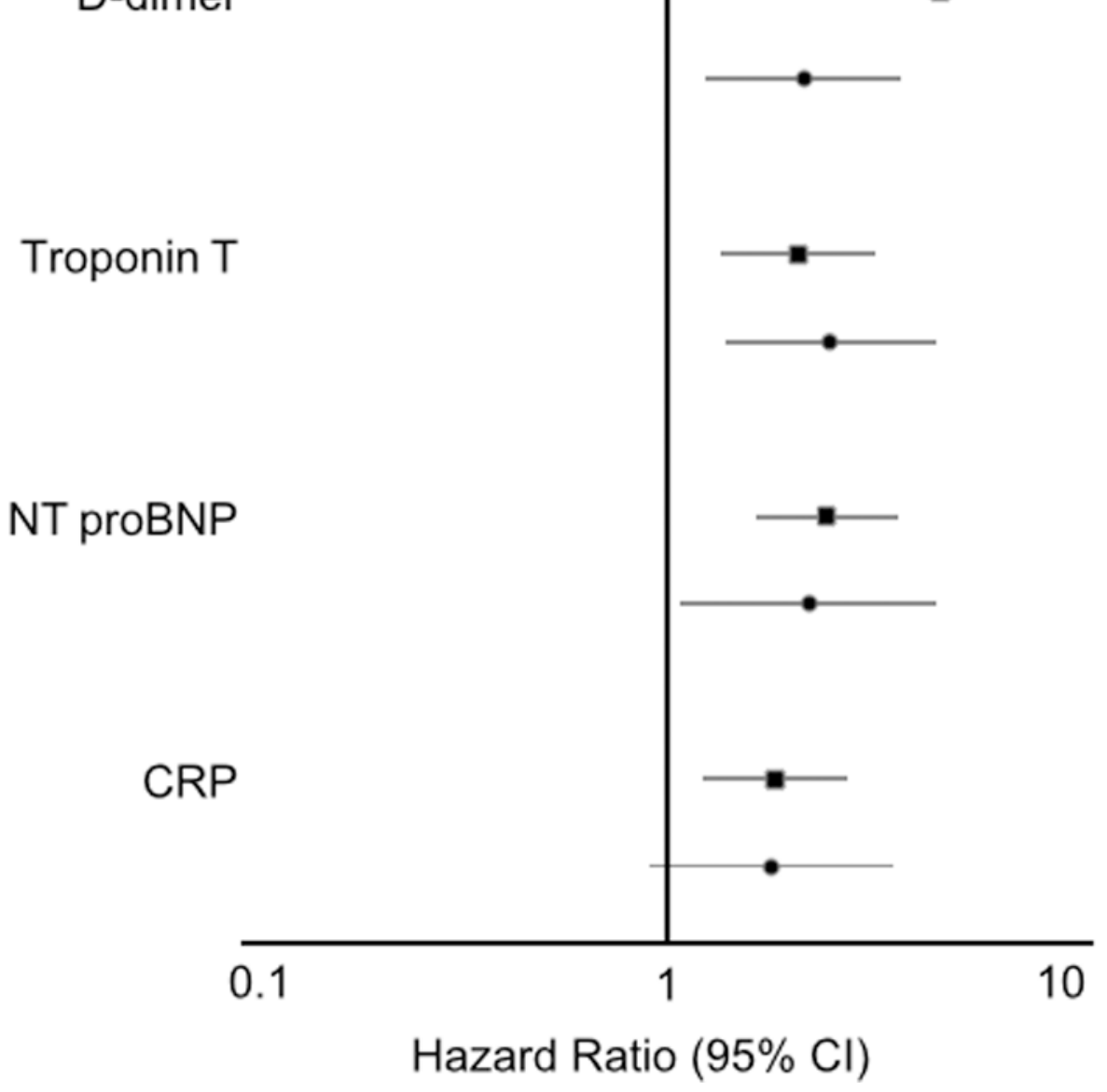

Figure 1.

Sex-specific hazard ratio* $(95 \% \mathrm{CI})$ of abdominal aortic aneurysm for participants in the highest compared to the lowest quartile of each biomarker, ARIC. *From separate sexspecific proportional hazard regressions (Model 2), considering each biomarker separately. 
WBC count

Fibrinogen

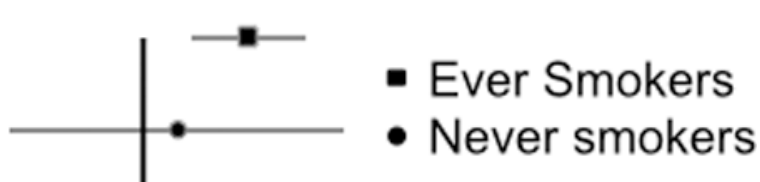

D-dimer

Troponin T

NT proBNP

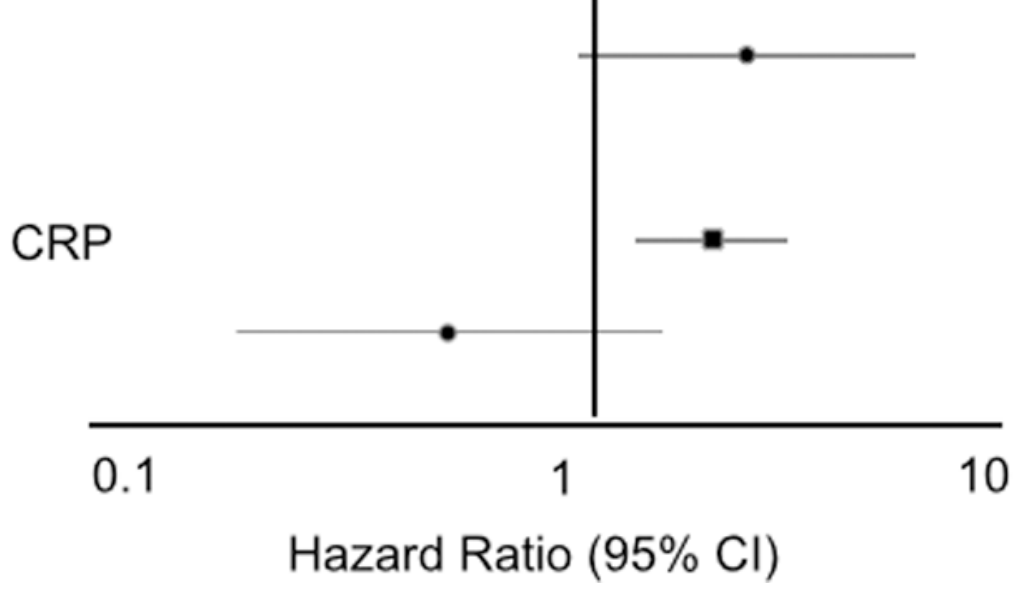

Figure 2.

Smoking-specific hazard ratio* $(95 \% \mathrm{CI}$ ) of abdominal aortic aneurysm for participants in the highest compared to the lowest quartile of each biomarker, ARIC. *From separate smoking-specific proportional hazard regressions (Model 2), considering each biomarker separately. 
Hazard Ratio $(95 \% \mathrm{Cl})$

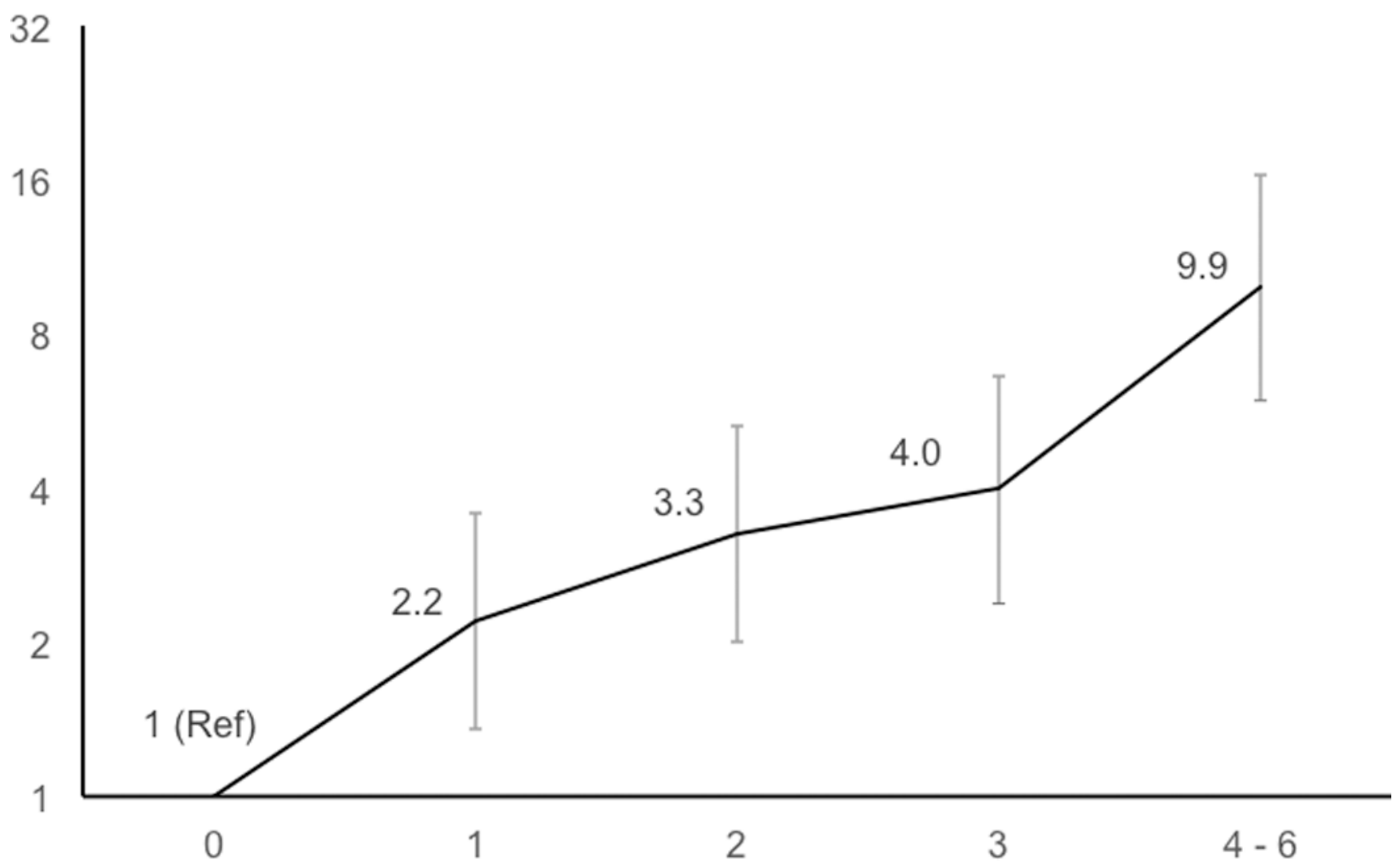

Number of Biomarkers in Highest Quartile

Figure 3.

Hazard ratio* $(95 \% \mathrm{CI})$ of abdominal aortic aneurysm after visit 4 according to the number of six biomarkers $\uparrow$ that participants had in the highest quartile, ARIC. *Based on Model 2. †WBC count, fibrinogen, D-dimer, troponin T, NT-proBNP, and CRP. 


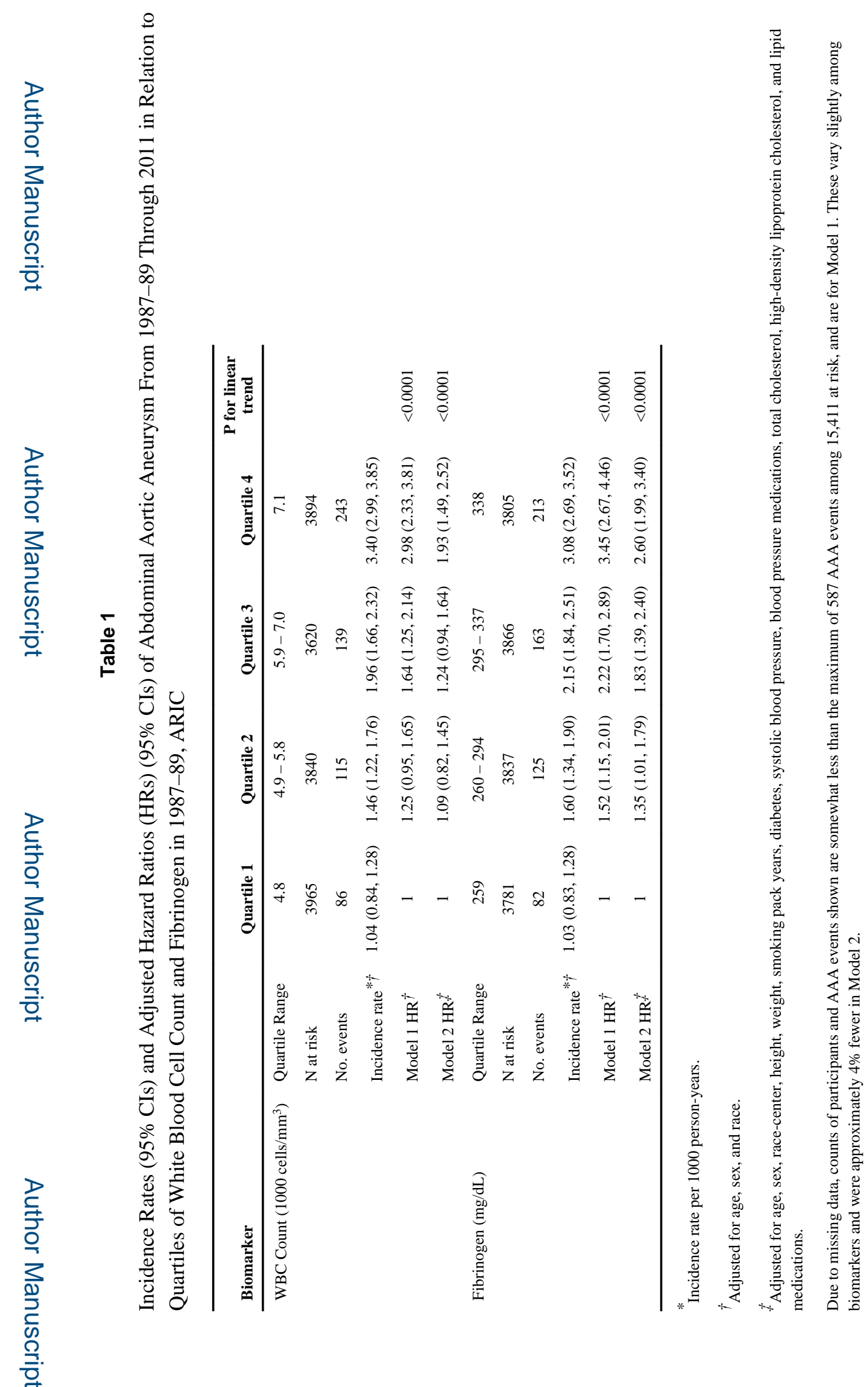

Circulation. Author manuscript; available in PMC 2016 August 18. 


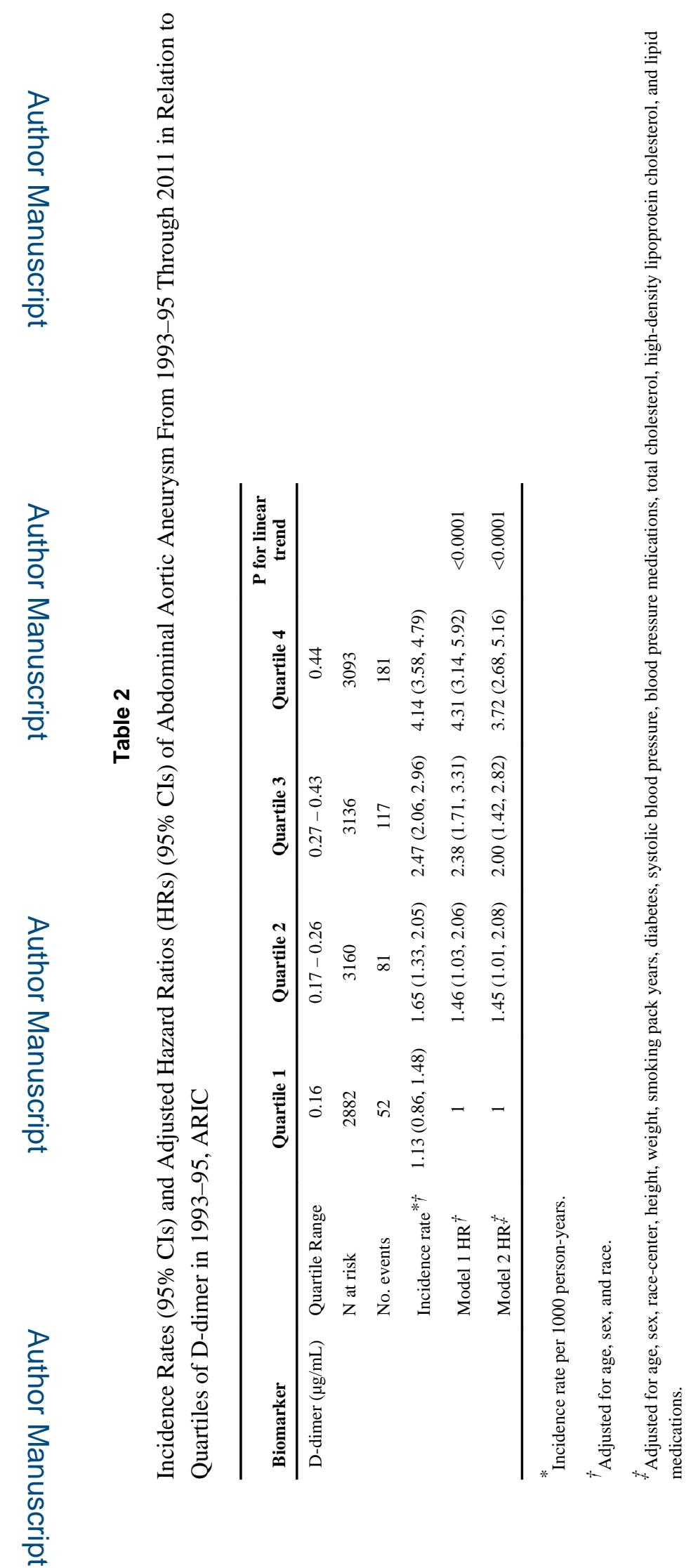

Circulation. Author manuscript; available in PMC 2016 August 18. 


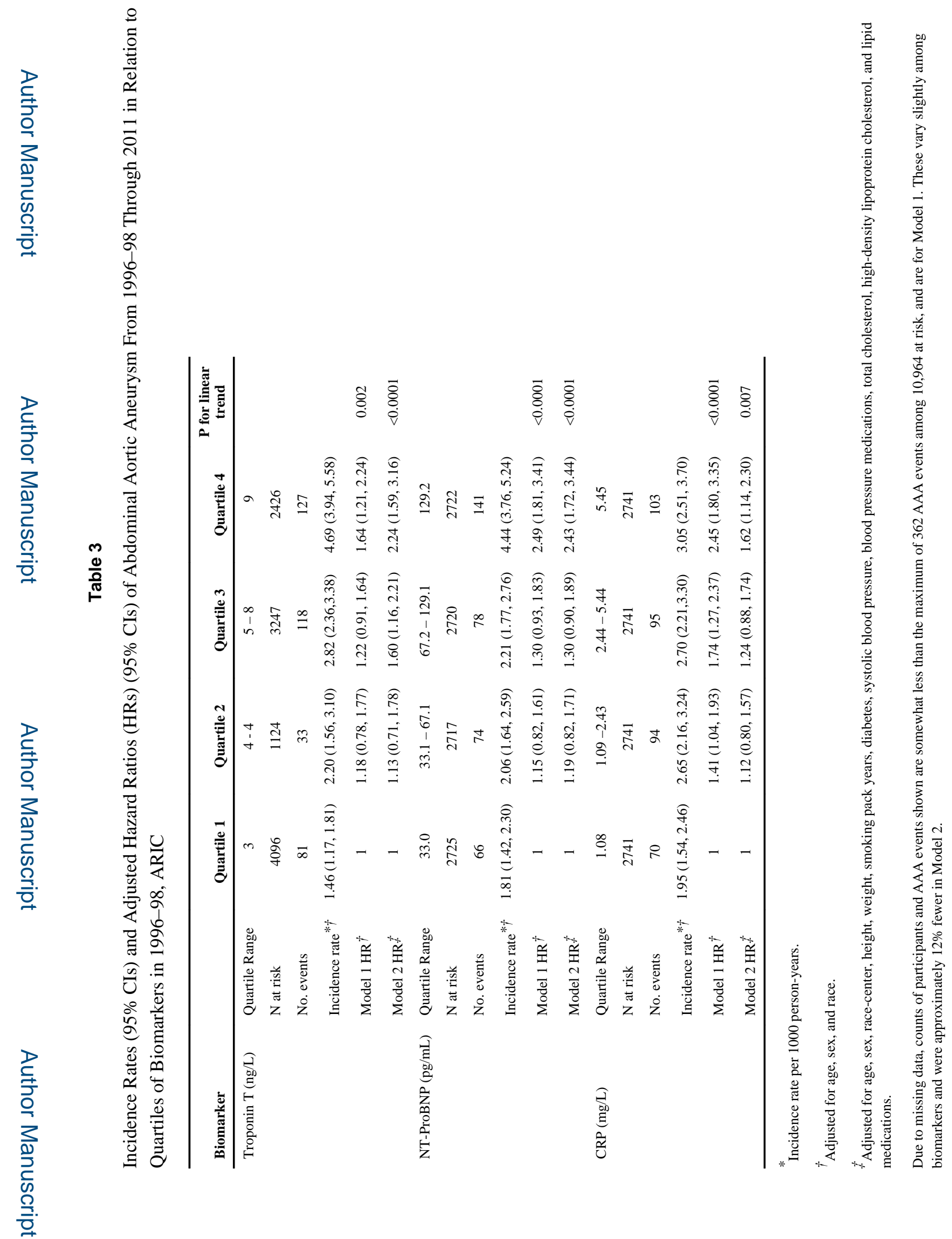

Circulation. Author manuscript; available in PMC 2016 August 18. 


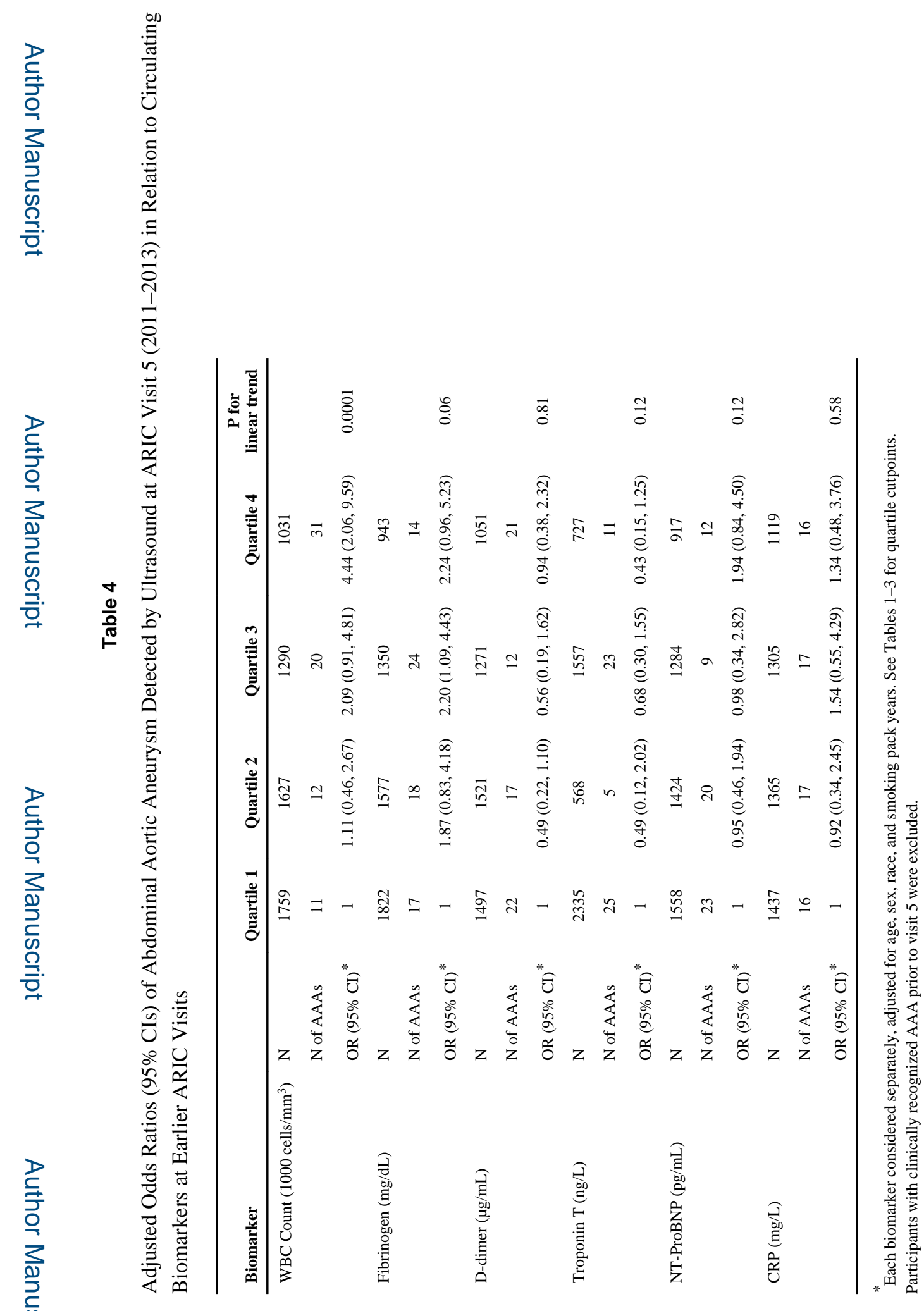

